\title{
Fluorescent dyes as a probe for the localized field of coupled surface plasmon-related resonances
}

\author{
M. Kreiter, T. Neumann, S. Mittler, and W. Knoll \\ Max Planck Institut für Polymerforschung, Ackermannweg 10, D - 55128 Mainz, Germany \\ J. R. Sambles \\ Department of Physics, University of Exeter, Exeter, Devon EX4 4QL, England
}

(Received 11 October 2000; published 20 July 2001)

\begin{abstract}
The fluorescence light of Cy5 dye molecules in the vicinity of a metal grating is studied for varying directions of both the exciting and the emitted light. A different angular dependence of the intensity of the emitted light is observed for different directions of excitation. Model calculations that take into account the localization of the electrical field of grating-coupled surface plasmon-related resonances are in good agreement with the experimental observations. In addition, the spatially inhomogenous photobleaching of the dye in the field of the coupled resonances is experimentally observed. These results can be viewed both as a way to use chromophores as molecular probes for the localized electrical near field of coupled surface plasmon-related resonances and as a way to manipulate dye molecules on a submicron scale.
\end{abstract}

DOI: 10.1103/PhysRevB.64.075406 PACS number(s): 42.25.Fx, 42.70.Qs, 68.49.Uv, 78.66.-w

\section{INTRODUCTION}

A periodicity at optical wavelengths leads to very specific optical properties of a surface. For example, for periodically modulated metallic surfaces, the incident light may interact with the surface-plasmon resonance, influencing strongly both the reflectivity of the surface and the optical near field. In this paper, the impact of these effects on the excitation and emission properties of a flourescing dye is investigated.

\section{A. The system under investigation}

The sample under investigation is sketched in Fig. 1. On top of a gold grating that is assumed as being infinitely thick, a thin dielectric film is deposited that contains the dye, followed by the ambient air. The periodically modulated metaldielectric interface is represented as a Fourier sum, specified by amplitudes $\mathrm{A}_{m}$ and phases $\phi_{m}$

$$
z(x)=\sum_{m=0}^{\infty} A_{m} \sin \left(m k_{g} x+\phi_{m}\right),
$$

where $z$ and $x$ are the Cartesian coordinates as illustrated in Fig. 2 and $\mathrm{k}_{g}$ is the modulus of the reciprocal grating vector that equals $2 \pi / \Lambda$, with $\Lambda$ being the grating pitch. The interface between the dielectric and air is assumed to have the same shape, being only shifted along the $z$ direction by the thickness of the dielectric film. The metal grating is illuminated by a plane electromagnetic wave, characterized by its wave-vector $\mathbf{k}_{\mathbf{i}}$. The polar angle $\theta$, the azimuthal angle $\psi$, and the wavelength $\lambda$ of the incident light can be varied in the experiment.

\section{B. Surface plasmons and coupled resonances}

Figure 3 shows the measured reflected intensity for TE polarized light as a function of the direction of the incident beam relative to a grating with a pitch of $\Lambda=770 \mathrm{~nm}$.

Clearly, two extended stripes of strongly reduced reflectivity can be observed. They can be attributed to the excitation of electromagnetic surface resonances. In a simple model (Ref. 1), the excitation of these surface resonances can be described as resonant coupling of the incident light to the surface-plasmon mode that has a fixed in-plane component $\mathbf{k}_{\mathbf{p}}$. Momentum matching to the in-plane component of a higher diffracted order of the incident wave-vector $\mathbf{k}_{\mathbf{i}, \mathbf{p}}$ requires

$$
\mathbf{k}_{\mathbf{p}}=\mathbf{k}_{\mathbf{i}, \mathbf{p}}+n \mathbf{k}_{\mathrm{g}},
$$

where $\mathbf{k}_{\mathbf{g}}$ is the reciprocal grating vector and $n$, the order of diffraction, an integer number. The subscript $i$ indicates that only the projection in the sample plane is considered. In Fig. 3 , an in-plane wave vector of $k_{p}=1.109 \mathrm{k}_{0}$ of the +1 st and -1 st order is indicated as a black line where $k_{0}$ is the modulus of the wave vector of the incident radiation in vacuum. Far away from $\psi=90^{\circ}$ the assignment of a fixed in-plane wave vector to the surface-plasmon resonance gives a good description of the observed position of the minima.

Close to $\psi=90^{\circ}$, the model of momentum matching breaks down as it can be seen by the deviation of the reflectivity minima from the lines of constant $\mathrm{k}_{p}$. This effect is referred to as a "photonic band gap" and has been first reported by Ritchie et al. ${ }^{2}$ It can be interpreted as follows: ${ }^{3}$ The plasmons that are simultaneously excited in +1 st and -1 st order are mutually coupled via the second-harmonic component of the grating. As a consequence, coupled resonances are supported by the grating that have in-plane wave vectors different from ordinary surface plasmons. Additionally, the width of these resonances is different from the unperturbed surface plasmon. Sarid ${ }^{4}$ has observed a similar phenomenon on a thin metal film. There, the interaction of the two surface plasmons on the two sides of the film led to



FIG. 1. Geometry of the sample. 




FIG. 2. Definition of the geometry for the reflection of a plane, monochromatic wave from a grating. The reciprocal grating vector, $\mathbf{k}_{\mathrm{g}}$ is lying in the plane of the grating surface, orthogonal to the grating grooves and has the modulus $2 \pi / \Lambda$ where $\Lambda$ is the grating period. The incident plane wave with wave-vector $\mathbf{k}_{\mathbf{i}}$ is specified by the following parameters: The polar angle $\theta$ between the wave vector and the surface normal and the azimuthal angle $\psi$ between the plane of incidence and the reciprocal grating vector $\mathbf{k}_{g}$.

the formation of two coupled resonances of different widths. In his paper, these resonances were labeled as "long-range surface plasmon" and "short-range surface plasmon," according to their propagation length. Although the mechanism of plasmon-plasmon coupling is different in our case, the resulting resonances exhibit different widths as well. For this reason, the terms "grating coupled long- (short-) range surface plasmon" (GLRP, GSRP) have been used to label the coupled resonances investigated in our work.

When the same experiment is performed with another wavelength, there is a similar reflectivity behavior. The only significant difference observed is a shift of the resonances in their polar angle $\theta$. This is demonstrated in Fig. 4 in a model calculation of the reflectivity of the identical grating structure when the illuminating light has a wavelength of $\lambda$ $=670 \mathrm{~nm}$. The calculation is based on a numerical algorithm that is described by Preist et al.,$^{5}$ further details about the modeling will be given below.

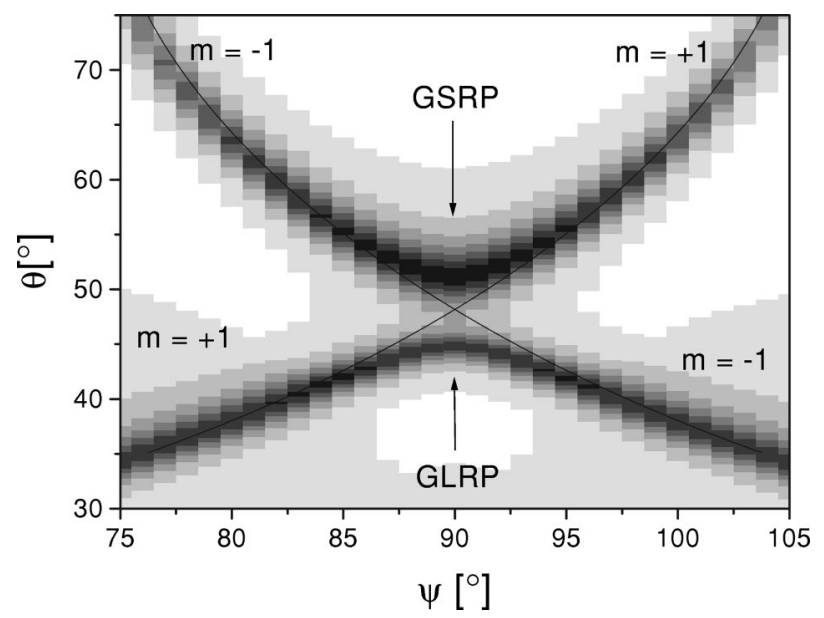

FIG. 3. Measured reflectivity of a gold grating $\Lambda=770 \mathrm{~nm}$ with a dielectric coating for TE-polarized light at $\lambda=632.8 \mathrm{~nm}$ as a function of the polar angle $\theta$ and the azimuthal angle $\psi$. The gray values represent the reflectivity, from white (1) to black (0). The black lines indicate constant values of the in-plane component of the first two diffracted orders.

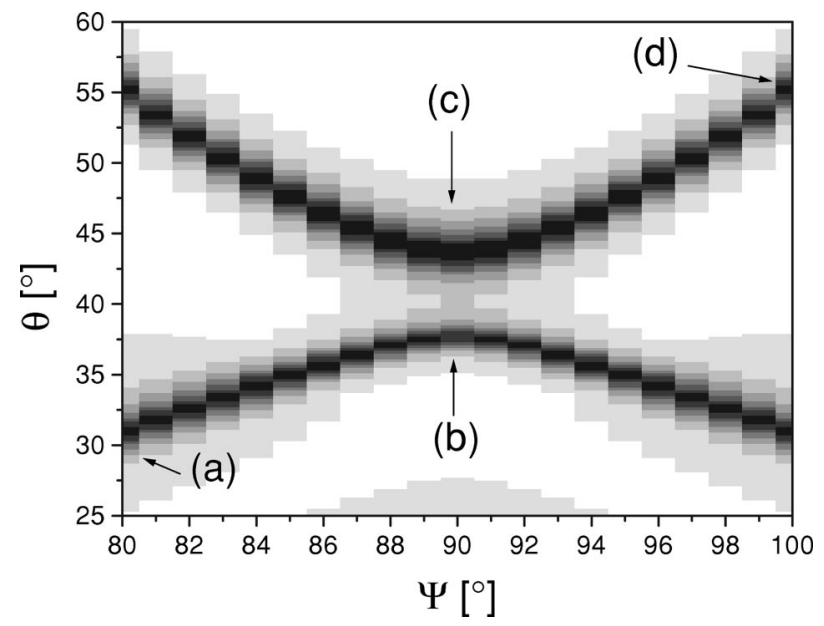

FIG. 4. Modeled reflectivity of a gold grating (details given in the caption of Fig. 8) with dielectric coating for TE-polarized light at $\lambda=670 \mathrm{~nm}$ as a function of polar angle $\theta$ and azimuthal angle $\psi$. The gray values represent the reflectivity, where white indicates maximum (1) and black a reflectivity of 0 . The letters indicate combinations of $\theta / \psi$ where field distributions were calculated.

\section{The electrical near field}

It is known that the coupling of surface plasmons does not only affect their in-plane wave vector, the electromagnetic near-field distribution is strongly affected as well. ${ }^{3}$ Model calculations suggest that coupled resonances are characterized by strongly localized electrical fields in the vicinity of the metal surface. Some analysis of the electrical fields is necessary to understand the interplay between fluorescing dyes and the electromagnetic surface resonances. The timeindependent electrical fields $\mathbf{E}$ are complex vector functions:

$$
\mathbf{E}(\mathbf{x})=\left(\begin{array}{c}
E_{x}(\mathbf{x}) e^{i \phi_{x}(\mathbf{x})} \\
E_{y}(\mathbf{x}) e^{i \phi_{y}(\mathbf{x})} \\
E_{z}(\mathbf{x}) e^{i \phi_{z}(\mathbf{x})}
\end{array}\right),
$$

where each Cartesian component of the electrical field vector $\mathbf{E}$ is given by its modulus and its phase. The averaged electrical field strength $\mathrm{E}$ is defined by

$$
[E(\mathbf{x})]^{2}=\left[E_{x}(\mathbf{x})\right]^{2}+\left[E_{y}(\mathbf{x})\right]^{2}+\left[E_{z}(\mathbf{x})\right]^{2} .
$$

Four typical electrical field distributions as determined by model calculations are shown in Fig. 5, chosen to represent the coupled resonances as well as the "freely" propagating surface plasmons. In Fig. 4, the selection of the chosen angles of incidence is indicated. Because the field is strongly reduced inside the metal, the grating profile appears as black contour.

Figures 5(b) and 5(c) correspond to the two coupled resonances. A strong lateral modulation of the strength of the electrical field along the grating surface is observed. By comparison of the two field distributions, one observes the coincidence of the minima of the GLRP (b) with the maxima of the GSRP (c) and vice versa. As is discussed in detail in Ref. 3, the second harmonic of the grating profile determines the positions of the maxima of the electric fields of the 




FIG. 5. Distributions of the electrical field strength in the vicinity of the grating surface when illuminated with TE polarized light at $\lambda=670 \mathrm{~nm}$. White corresponds to high, black to vanishing field strength. The field distributions correspond to (a) $\psi=80^{\circ}$, $\theta=31.1^{\circ} \quad(m=+1)\left(\right.$ b) $\psi=90^{\circ}$, $\theta=37.7^{\circ},(\mathrm{GLRP})(\mathrm{c}) \psi=90^{\circ}, \theta$ $=43.7^{\circ},($ GSRP $)(\mathrm{d}) \psi=100^{\circ}, \theta$ $=55.1^{\circ},(m=+1)$. coupled resonances. The positions of maximum field strength of the GSRP coincide with the maxima of the second harmonic of the grating profile while the GLRP has its maximum electrical field in the minima.

For the "normal" surface plasmons outside the band-gap region [Figs. 5(a) and 5(d)], some lateral variation in the electrical field strength is visible, but it is much weaker and no correlation of the maximum position with the second harmonic of the grating profile is observed. This is the expected behavior for surface plasmons, propagating like twodimensional light waves along the interface.

The contrast between free-surface plasmons and coupled resonances can be seen more clearly in Fig. 6, where the electrical field distribution along a line $10 \mathrm{~nm}$ above the metal surface (which is right in the middle of the dielectric layer) is plotted. Neglecting some perturbation at $x=650 \mathrm{~nm}$ (right in the gratings minimum), the two coupled resonances show electrical field distributions that are close to sinusoidal and phase-shifted by $180^{\circ}$ relative to each other. The correlation with the second-harmonic component of the grating profile (thick full curve in Fig. 6) is clearly seen. The other two distributions, corresponding to free-surface plasmons show much less variation in intensity and the correlation to the maximum/minimum positions of the second harmonic of the grating profile is very weak.

This shows that by adjusting the angle of incidence, an experimental parameter that can be easily varied, it is possible to set up high electrical field strengths that have a characteristic lateral distribution. They show a modulation that has half the wavelength of the fundamental period of the grating.

\section{Fluorescence and surface plasmons}

It is well known that both the excitation and emission properties of fluorescent dyes are strongly influenced by the existence of the surface-plasmon resonance. ${ }^{6-9}$ The electrical field in the vicinity of a metal surface is considerably enhanced upon excitation of the surface-plasmon resonance. For gold at a wavelength of $\lambda=632.8 \mathrm{~nm}$ the intensity on the metal surface is roughly 20 times higher than in the exciting plane wave. As a consequence, the excitation probability of a chromophore is enhanced by the same factor.

This has been shown by Knobloch et al. ${ }^{10}$ for grating coupling to the surface-plasmon resonance. In this experiment, a significant increase of fluorescence intensity from a silver surface was observed when the incident light excites the

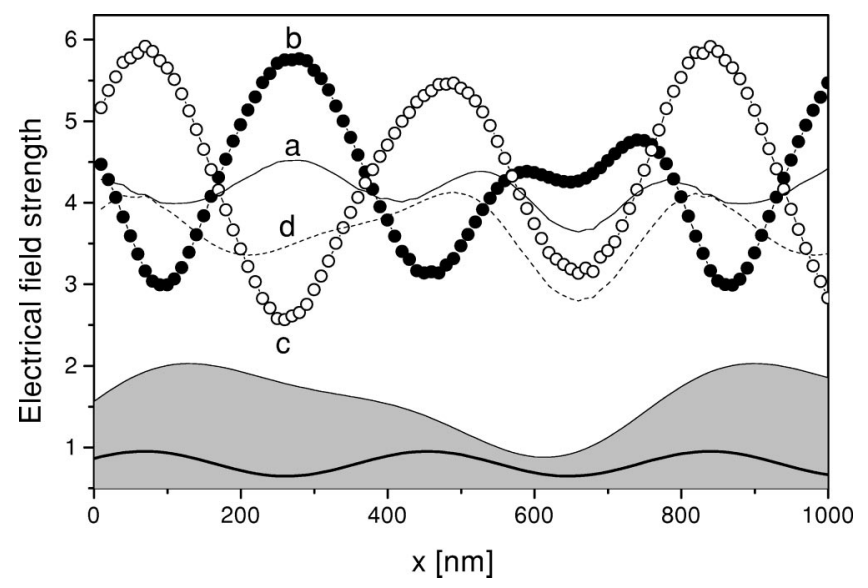

FIG. 6. Electrical field strengths along a line at constant distance of $10 \mathrm{~nm}$ above the metal surface for the four cases depicted in Fig. 4 (a) $\psi=80^{\circ}, \theta=31.1^{\circ}$, straight line, free-surface plasmon $(m=+1)$ (b) $\psi=90^{\circ}, \theta=37.7^{\circ}$, full circles, (GLRP) (c) $\psi=90^{\circ}$, $\theta=43.7^{\circ}$, open circles, (GSRP) (d) $\psi=100^{\circ}, \theta=55.1^{\circ}$, dashed line, free-surface plasmon $(m=+1)$. The entire grating profile is indicated as a gray area graph, the thick line represents its secondharmonic component. 
surface-plasmon resonance compared to reflecting geometries where no resonant excitation occurs.

More investigations on a grating structure in silver were performed by Kitson et al. ${ }^{11}$ and Andrews et al. ${ }^{12}$ Here, the polar angle and the wavelength of the excitation beam were varied, resulting in a band-gap structure in the reflectivity similar to the one shown in Fig. 3. It was observed that the emission intensity of the dye exhibits the same band-gap structure as the reflectivity. This proves that both coupled resonances and free-surface plasmons effectively enhance the excitation probability of a fluorophore.

Not only the excitation probability depends on the dielectric environment of the dye molecule. According to Fermi's golden rule (compare, e.g., Ref. 13), the emission probability $P_{e m}$ of a chromophore from the excited state $m$ to the ground-state $k$ depends both on the intrinsic properties of the molecule and on the surrounding.

$$
P_{e m}=\frac{4 \pi^{2}}{h}\left|V_{m k}\right|^{2} \sigma_{m k}
$$

The matrix element $V_{m k}$ gives the intrinsic probability of the emitter to relax from the excited-state $m$ into the groundstate $k$. The influence of the surrounding on the emission characteristics comes in via $\sigma_{m k}$, the "density of states" or number of decay channels for the emission process. This number can be obtained from a purely classical treatment by regarding the emitting molecule as a point dipole. The integrated intensity of the classical electromagnetic flux away from the molecule determines the probability that a photon is emitted.

A simple geometry where the influence of the number of decay channels on the emission characteristics has been studied extensively in theory ${ }^{14-16}$ and experiment are one or more plane interfaces in front of which the excited dipole is placed. This work is comprehensively treated in a review article by Barnes. ${ }^{17}$ A single metal surface ${ }^{18}$ provides a good illustration. The surface-plasmon resonance leads to a significant enhancement of decay channels (density of states) for dye molecules that are close enough to the interface to transfer their energy efficiently to the surface plasmon. This is reflected in a drastically reduced lifetime of the excited state when the emitter is placed closer to the interface than about $20 \mathrm{~nm}$. The first experimental proof that the surface plasmon as an intermediate state plays an important role for the coupling of the excitiation energy of a chromophore to light was given in the early 1980's. ${ }^{19,20}$ These authors observed the fluorescence intensity generated on a dye-coated silver grating upon excitation at a fixed angle as a function of the direction of the emission. Pronounced peaks in the emission intensity were found that were due to processes that involve the excitation of the surface-plasmon resonance as an intermediate step. This was proven by showing that the directions of enhanced emission could be reproduced by applying the momentum matching condition Eq. (1.2) to the emitted light.

Kitson et al. ${ }^{21}$ extended the observation of surfaceplasmon-enhanced emission of fluorescence light to the complete half sphere above the grating, including the case $\psi$ $\neq 0^{\circ}$. Especially, experimental geometries were investigated in Ref. 22, for which the emission process takes place via coupled resonances (GLRP, GSRP). In all of these experiments, emission maxima were observed in directions corresponding to reflectivity minima in a reflection experiment performed with the emission wavelength. This includes the formation of a band-gaplike structure in the emission intensity recorded over the entire half sphere above the grating.

The investigations presented in this paper are a somewhat natural extension of the investigations reviewed above: both the excitation and the emission of the dye molecules will involve the surface-plasmon resonance as an intermediate step. This will yield new insights that could not be obtained by having surface plasmons involved in only one of these two processes. Especially the field intensities of coupledsurface resonances vary strongly along the interface (See Fig. 6). This implies that the excitation probability of a molecule close to the surface depends strongly on its position along the surface of the grating. So, in a simplified view, there are two sets of chromophores: the ones being placed in the field maxima of the GLRP, the other ones are placed in the field maxima of the GSRP. It is possible to excite, selectively, one of the two sets of chromophores by choosing the appropriate excitation angle.

If the optimum excitation angle of a certain molecule depends on its position on the grating, it should be expected that the same holds for the angle under which the strongest emission can be observed. Considering a molecule positioned right in the intensity maximum of the GLRP, the coupling to this surface resonance will be stronger than to the GSRP. Therefore, this excited molecule is expected to lose its energy predominantly via the GLRP. Which surface resonance is involved in the outcoupling process can be determined by recording the emission direction of the fluorescence. The concept presented above allows for a selective excitation of only a fraction of the molecules at the interface, as well as selective fluorescence read out. Since the two groups of dye molecules are defined as the ones being positioned either in the maxima or in the minima of the 2nd harmonic of the grating profile, their lateral distance is very small (less than $200 \mathrm{~nm}$ from maximum to minimum excitation in the case investigated here.)

\section{CALCULATION OF FLUORESCENCE INTENSITY AS A FUNCTION OF THE EXPERIMENTAL GEOMETRY}

In this section, a model is presented that allows for the calculation of the fluorescence intensity of molecules being randomly distributed within the polymer layer on top of the grating as depicted schematically in Fig. 1. The direction of the exciting laser beam and the direction of the emitted light are free parameters of the model. First, the response is calculated for one single molecule. Then, the expected fluorescence intensity is obtained by an appropriate averaging procedure.

In our experiments, a two-step process has to be considered for each molecule. First, the excitation probability for a given molecule at the position $\mathbf{x}$ with the transition dipole moment for the excitation $\mathbf{r}_{\mathbf{e x}}$ must be determined. The ex- 
citing light wave is specified by a fixed wavelength $\lambda_{e x}$, the direction of incidence, given by the solid angle $\Omega_{e x}$ and its polarization. $\Omega_{e x}$ is used here as an abbreviation for $\left(\Theta_{e x}, \Psi_{e x}\right)$. The absorption process is described as the excitation of an electrical dipole with a fixed oscillation axis by the incident electrical field. The detailed theory of this model can be found in the literature, e.g., Ref. 13. Essentially, the transition probability for the excitation process $\mathbf{P}_{e x}$ is calculated by using Fermi's golden rule [Eq. (1.5)]. For a fixed direction of the dipole, the transition matrix element is given as

$$
V_{m k}=-\mathbf{r}_{\text {ex }} \cdot \mathbf{E},
$$

with $\mathbf{E}$ being the (time-independent) electrical field. Therefore, the probability for the excitation $P_{e x}$ will have the form

$$
P_{e x}\left(\mathbf{x}, \mathbf{r}_{\mathrm{ex}}, \Omega_{e x}\right) \propto\left[\mathbf{E}\left(\mathbf{x}, \Omega_{e x}\right) \mathbf{r}_{\mathrm{ex}}\right]\left[\mathbf{E}\left(\mathbf{x}, \Omega_{e x}\right) \mathbf{r}_{\mathrm{ex}}\right]^{*},
$$

where the asterisk denotes the complex conjugate. The second step that must be modeled theoretically is the emission process. A complete treatment of the problem would consist of calculating the electrical field of the oscillating dipole with the appropriate boundary conditions imposed by the grating. From this, the probability that the light is emitted into a certain solid angle (the detector) could be derived. Additionally, the photonic energy that is dissipated in the gold substrate could be tracked. But since no mathematical algorithms are known to solve this problem, this exact method cannot be used.

For this reason, a simpler approach is chosen to calculate the probability that the emitter radiates its energy into a certain solid angle. It is based on viewing the dye molecule and the detector as two systems that may exchange energy by a photon. The direction of the energy exchange that is of interest, from the dye molecule to the detector cannot be directly calculated. The reverse direction would be an emitter at the detector position transferring its energy to the dye molecule at the grating surface. This problem has already been solved in the previous section and is easily adapted by changing the wavelength of the radiation to the emission wavelength of the fluorescent molecule. Because the coupling strength is equal for both directions of the energy transfer, the problem is solved. The following equation gives the relative probability $P_{e m}$ for a given emitting dipole to radiate its energy into a certain direction:

$$
P_{e m}\left(\mathbf{x}, \mathbf{r}_{\mathrm{em}}, \Omega_{e m}\right) \propto\left[\mathbf{E}\left(\mathbf{x}, \Omega_{e m}\right) \mathbf{r}_{\mathrm{em}}\right]\left[\mathbf{E}\left(\mathbf{x}, \Omega_{e m}\right) \mathbf{r}_{\mathrm{em}}\right]^{*},
$$

where the vector $\mathbf{r}_{\mathrm{em}}$ denotes the transition dipole moment for the emission process and $\Omega_{e m}$ the solid angle of detection.

One disadvantage of this approach should be mentioned here. All information about decay channels that are not ultimately transferred into plane light waves is lost, therefore, it is impossible to give absolute probabilities that the excitation energy of the dye molecule will be transformed into a photon, propagating in a certain direction. As long as it can be assumed that the density of alternative decay channels does not vary quickly along the grating surface, this approach is applicable for the approximate calculation of an emission pattern, where only intensities at different emission directions are compared.

The next step consists of taking the average of all molecules that are involved. It is assumed that they are randomly distributed within the polymer layer and randomly oriented. The probability that fluorescence light is detected in the emission direction $\Omega_{e m}$ upon irradiation under the excitation direction $\Omega_{e x}$ will be an average over all molecules. First, the the different orientations of the molecules are evaluated. If the transition dipole moments for excitation and emission coincide and the molecules do not rotate between absorption and emission, the directional average has to be taken over the combined process as well ("fixed dipole model"). In this case, the directionally averaged probability for the combined excitation-emission process, $P_{d a}$ is given by

$$
P_{d a}\left(\Omega_{e x}, \Omega_{e m}, \mathbf{x}\right) \propto\left\langle P_{e x}\left(\Omega_{e x}, \mathbf{x}, \mathbf{r}\right) P_{e m}\left(\Omega_{e m}, \mathbf{x}, \mathbf{r}\right)\right\rangle,
$$

where the brackets denote the averaging and the vector $\mathbf{r}$ is the (common) direction of the transition dipole moment. Inserting Eqs. (2.2) and (2.3) yields

$$
\begin{aligned}
P_{d a}\left(\Omega_{e x}, \Omega_{e m}, \mathbf{x}\right) & \\
\propto & \left|E_{x}^{e x}\right|^{2}\left[\frac{3}{8}\left|E_{x}^{e m}\right|^{2}+\frac{1}{8}\left|E_{y}^{e m}\right|^{2}+\frac{1}{8}\left|E_{z}^{e m}\right|^{2}\right] \\
& +\left|E_{y}^{e x}\right|^{2}\left[\frac{1}{8}\left|E_{x}^{e m}\right|^{2}+\frac{3}{8}\left|E_{y}^{e m}\right|^{2}+\frac{1}{8}\left|E_{z}^{e m}\right|^{2}\right] \\
& +\left|E_{z}^{e x}\right|^{2}\left[\frac{1}{8}\left|E_{x}^{e m}\right|^{2}+\frac{1}{8}\left|E_{y}^{e m}\right|^{2}+\frac{3}{8}\left|E_{z}^{e m}\right|^{2}\right] \\
& +\frac{1}{2}\left[\left|E_{x}^{e x} E_{y}^{e x}\right|\left|E_{x}^{e m} E_{y}^{e m}\right|\right. \\
& +\left|E_{x}^{e x} E_{z}^{e x}\right|\left|E_{x}^{e m} E_{z}^{e m}\right| \\
& \left.+\left|E_{y}^{e x} E_{z}^{e x}\right|\left|E_{y}^{e m} E_{z}^{e m}\right|\right] .
\end{aligned}
$$

In this equation, the dependence of the electrical fields on $\mathbf{x}, \Omega_{e x}$, and $\Omega_{e m}$ is not explicitly written for readability.

If the transition dipole moment is different for the excitation and the emission process, either on the molecular level or due to rotation of the chromophore, the average over orientations must be taken separately. One model is based on the assumption that there is no correlation between the transition dipole moments of excitation and emission process. Because this model would be valid for quickly and freely rotation dipoles, it is called "rotating dipole model." In this case, the directional averages must be calculated independently, therefore, the emission probability equals

$$
\begin{aligned}
& P_{d a}\left(\Omega_{e x}, \Omega_{e m}, \mathbf{x}\right) \\
& \quad \propto \sum_{\mathbf{x}} P_{d a}\left\langle P_{e x}\left(\Omega_{e x}, \mathbf{x}, \mathbf{r}\right)\right\rangle_{\mathbf{r}_{\mathbf{e x}}}\left\langle P_{e m}\left(\Omega_{e m}, \mathbf{x}, \mathbf{r}\right)\right\rangle_{\mathbf{r}_{\mathbf{e m}}}
\end{aligned}
$$


With Eqs. (2.2) and (2.3), this yields

$$
P_{d a}\left(\Omega_{e x}, \Omega_{e m}, \mathbf{x}\right) \propto \frac{1}{4}\left|\mathbf{E}\left(\Omega_{e x}, \mathbf{x}\right)\right|^{2}\left|\mathbf{E}\left(\Omega_{e m}, \mathbf{x}\right)\right|^{2} .
$$

Finally, these probabilities are spatially averaged over all possible positions of the dye molecule in order to obtain the fluorescence intensity $I$ for a given direction of excitation and emission

$$
I\left(\Omega_{e x}, \Omega_{e m}\right) \propto \frac{\sum_{\mathbf{x}} P_{d a}\left(\Omega_{e x}, \Omega_{e m}, \mathbf{x}\right)}{N},
$$

where $N$ denotes the number of points where the above equations are evaluated. A numerical modeling routine ${ }^{5}$ allows for the determination of the electrical field distributions $\mathbf{E}(\Omega, \mathbf{x})$. The determination of the set of parameters that is suited for the proper description of the sample under investigation will be described in Sec. IV A.

\section{EXPERIMENT}

\section{A. Sample preparation}

Based on initial calculations, gratings with a pitch of roughly $\Lambda=750 \mathrm{~nm}$ and an amplitude of the first-harmonic component of the surface profile of $A_{1}=20-30 \mathrm{~nm}$ turned out to be best suited for our investigations. Additionally, a strong blazing of the structure is necessary to get significant coupling to both branches of coupled surface resonances because on symmetric gratings, optical excitation is possible for one resonance only. ${ }^{23}$

A photoresist film (Shipley microposit) with a thickness of $50 \mathrm{~nm}$ was deposited by spin-coating on a fused silica substrate. A grating was written holographically ${ }^{24}$ into the resist. Subsequently, the grating was transferred into the substrate by reactive ion beam etching with a mixture of $\mathrm{O}_{2}$ and $C F_{4}$. First, the sample was exposed to a normally incident ion beam for one minute (corresponding to the ablation of 20 $\mathrm{nm}$ photoresist and $40 \mathrm{~nm}$ fused silica) then, the sample was tilted by $75^{\circ}$, resulting in a highly asymmetric etching that was continued for another eight minutes to ensure complete removal of the resist. By thermal evaporation in a commercial evaporation chamber (Balzers), the sample was covered with an optically thick $(150 \mathrm{~nm})$ gold film. A thin, thermally evaporated chromium film between glass and gold was used for better adhesion between the two materials.

A thin film of poly-(vinyl alcohol) with the chromophore Cy5, bound to streptavidin (obtained from Amersham Pharmacia), as a guest-host system was spin coated out of aqueous solution (containing one weight per cent polymer) at 4000 RPM. Cy5 may be excited by light with a wavelength of $\lambda_{e x}=632.8 \mathrm{~nm}$ and has an emission maximum at $\lambda_{e m}$ $=670 \mathrm{~nm}$. For these experiments, the streptavidin molecule is just used as an anchor for the dye, its chemistry is of no importance.

\section{B. Optical setup}

The grating is mounted in $\psi_{e x}=90^{\circ}$ geometry on the setup that is sketched in Fig. 7. The beam of a HeNe Laser

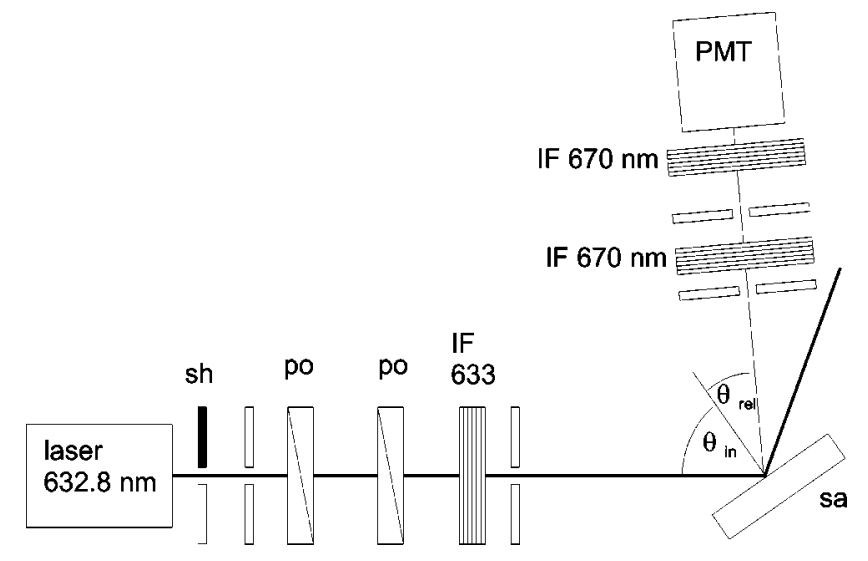

FIG. 7. Schematic experimental setup: The abbreviations stand for shutter (sh), polarizer (po), interference filter (IF), sample (sa), and photomultiplier tube (PMT).

$(\lambda=632.8 \mathrm{~nm})$ is attenuated and fixed to TE-polarization by two polarizers. Right in front of the sample, all frequency components in the laser beam but the fundamental are removed with an interference filter $(633 \mathrm{~nm})$. Stray light from the optical path is minimized with the aid of pinholes. A shutter blocks the laser beam automatically when no data are taken in order to minimize photobleaching of the dye molecules as far as possible. All components are mounted on an optical bench.

The incident beam intersects the axis of a goniometer, which is normal to the drawing plane. There, the sample is mounted with the grating grooves lying in the plane of incidence and the goniometer axis lying in the sample surface $\left(\psi_{e x}=90^{\circ}\right.$ geometry). This allows for the variation of $\theta_{e x}$, the polar angle of the excitation beam.

On the detection axis, the fluorescence intensity generated on the sample surface is measured as a function of its direction: first, a pinhole (diameter $2 \mathrm{~mm}$ ) determines the solid angle relative to the sample that is investigated. Its distance to the sample being $150 \mathrm{~mm}$ leads to an angular resolution of $0.76^{\circ}$. An interference filter $(670 \mathrm{~nm})$ removes all frequency components in the light except the one corresponding to the wavelength of the emission maximum of the dye. A pinhole reduces stray light before the light reaches the photomultiplier tube. All these components are mounted together rigidly and the entire detection unit is mounted on a second (detector) goniometer with an axis that coincides with the one of the sample goniometer. The detection unit can be moved vertically (normally to the plane of the drawing in Fig. 7) in order to investigate $\psi_{e m}$ other than $90^{\circ}$. In this case, the optical axis of the detection system still has to point towards the sample. The required tilting is established with the aid of a ball joint that links the detection system to an optical bench that is screwed on the detector goniometer. The direction of emission that is investigated in this case is defined by the angle between the axis of the detection system and the plane of incidence, $\psi_{\text {tilt }}$ :

$$
\psi_{\text {tilt }}=\arctan \left(\frac{h}{d}\right)
$$


with the height $h$ of the first pinhole over the plane of incidence and the distance $d$ that is the projection of the detection axis onto the plane of incidence. As a second degree of freedom the detector can be moved on a circle around the sample by the detector goniometer. Its position is determined by the relative polar angle $\theta_{\text {rel }}$ that is defined as the angle between the surface normal of the sample and the projection of the detection axis onto the plane of incidence. It should be mentioned here that $\theta_{r e l}$ and $\psi_{t i l t}$, which can be varied with the experimental setup, are not equal to the polar angle $\theta_{e m}$ and the azimuthal angle $\psi_{e m}$, which are introduced in Fig. 2 and are the basis for the theoretical treatment. By basic trigonometry it can be shown that

$$
\begin{gathered}
\tan \left(\theta_{e m}\right)=\frac{\left\{\left[\sin \left(\psi_{t i l t}\right)\right]^{2}+\left[\sin \left(\theta_{r e l}\right) \cos \left(\psi_{t i l t}\right)\right]^{2}\right\}^{1 / 2}}{\cos \left(\psi_{t i l t}\right) \cos \left(\theta_{r e l}\right)} \\
\tan \left(\psi_{e m}+90^{\circ}\right)=\frac{\sin \left(\psi_{t i l t}\right)}{\sin \left(\theta_{r e l}\right) \cos \left(\psi_{t i l t}\right)}
\end{gathered}
$$

These relations allow a comparison between model calculation and experiment. Note that for detection in the plane of incidence $\left(\psi_{t i l t}=0^{\circ}\right)$, the above expressions reduce to $\psi_{e m}$ $=90^{\circ}, \theta_{\text {em }}=\theta_{\text {rel }}$.

For the optical characterization of the grating, the specularly reflected laser light is recorded with a photodiode that is mounted on the detection axis instead of the components that were used to record the fluorescence. Upon variation of the polar angle of the incident laser beam $\theta_{i n}$ the intensity that is reflected from the grating is recorded. These data are normalized to the intensity of the incident beam that is measured by removing the sample and adjusting the detector to collect all the transmitted light.

During the measurement there was some bleaching of the dye. For the series of measurements where different tilting angles $\psi_{\text {tilt }}$ were investigated (compare Sec. IV D), this effect was too pronounced to be neglected. During one single measurement, (variation of $\theta_{e x}$ ) the observed decrease in intensity was less than $10 \%$ as determined by reference measurements before and after. The data were corrected for this effect assuming a linear decrease in intensity with time. Subsequent measurements were normalized using the appropriate reference signal.

It is a problem that this correction routine does not account for the fact that the bleaching rate of the molecules is strongly site dependent as will be explored in Sec. IV E. For that reason, care must be taken that the bleaching is not too strong in order to obtain useable data.

\section{RESULTS}

\section{A. Sample characterization with reflectivity measurements}

The basis of the calculations of the local fields that are experienced by the fluorescing molecules is a set of parameters which describes the optical response of the grating: the shape of the grating, the dielectric constant of the gold, as

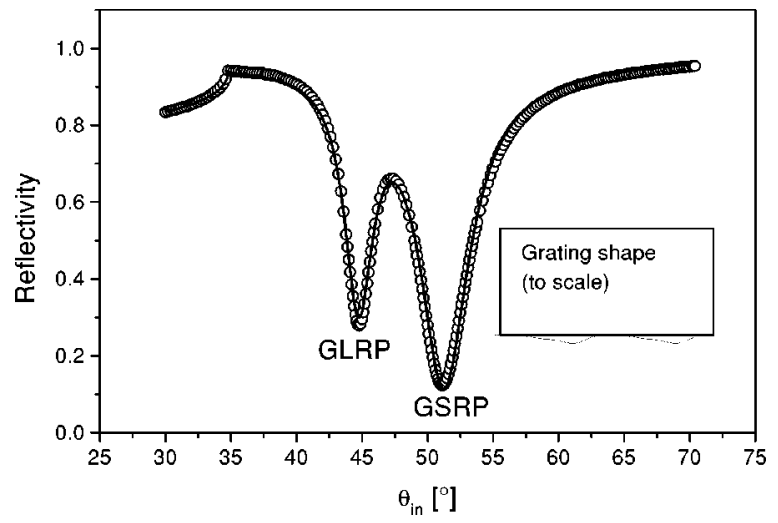

FIG. 8. Reflectivity in $\psi=90^{\circ}$ geometry with the incident light $(\lambda=632.8 \mathrm{~nm})$ being TE-polarized. The straight line is a model calculation based on the parameter set: $\Lambda=770.35 \mathrm{~nm}, \mathrm{~A}_{1}$ $=25.6 \mathrm{~nm}, \mathrm{~A}_{2}=7.62 \mathrm{~nm}, \phi_{2}=25.6^{\circ}, \mathrm{A}_{3}=3 \mathrm{~nm}, \phi_{3}=63^{\circ}, \epsilon_{A u}$ $=-11.2+i 1.17$, polymer layer with thickness $19.1 \mathrm{~nm}$ and dielectric constant $\epsilon=2.25$. The grating shape as determined from the fit is shown in the inset.

well as the thickness and the refractive index of the polymer film. Reflectivity measurements are best suited to obtain these parameters. ${ }^{25,26}$

Here, measurements of the $\theta$-dependent reflectivity in the $\psi=90^{\circ}$-geometry, both of the bare gold surface and the multilayer system with polymer film allow for the determination of the grating profile and dielectric constants by a least squares fit to the data.

Their values are given in the figure caption of Fig. 8, which shows calculated and experimental data as obtained with the dielectric film.

For the model calculations at a wavelength of $\lambda$ $=670 \mathrm{~nm}$, the dielectric constant of the gold was extrapolated from the value that was found for $\lambda=632.8 \mathrm{~nm}$ according to a linear extrapolation procedure that was discussed elsewhere [Kre99]. An $\epsilon(\lambda=670 \mathrm{~nm})=-13.84$ $+i 1.053$ was used.

\section{B. Fluorescence emission in the plane of incidence}

The fluorescence light was measured as a function of $\theta_{\text {rel }}$ for the angles of incidence $\theta_{e x}=44.8^{\circ}$ and $\theta_{e x}=51^{\circ}$, with $\psi_{e x}=90^{\circ}$ and $\psi_{t i l t}=0^{\circ}$. This is the same excitation geometry as used for the reflectivity scan in Fig. 8. From there, it can be seen that the two excitation angles $\theta_{e x}$ were chosen right in the two reflectivity minima, assuring an effective excitation of the two coupled resonances (GLRP, GSRP).

The emitted intensity as a function of the emission angle $\theta_{e m}$ is shown in Fig. 9. Two clear maxima are observed at $\theta_{\text {rel }}=37.5^{\circ}$ and $\theta_{r e l}=43.5^{\circ}$. The positions where the minima are observed in a reflection experiment with the corresponding wavelength $\lambda_{e m}=670 \mathrm{~nm}$ (compare the model calculation in Fig. 4) coincide well with the positions of these emission peaks. Therefore, they can be attributed to an enhanced emission that is mediated by the two coupled resonances at $\lambda_{e m}=670 \mathrm{~nm}$.

An important observation is made regarding the relation between the maximum values of the two peaks for the two 


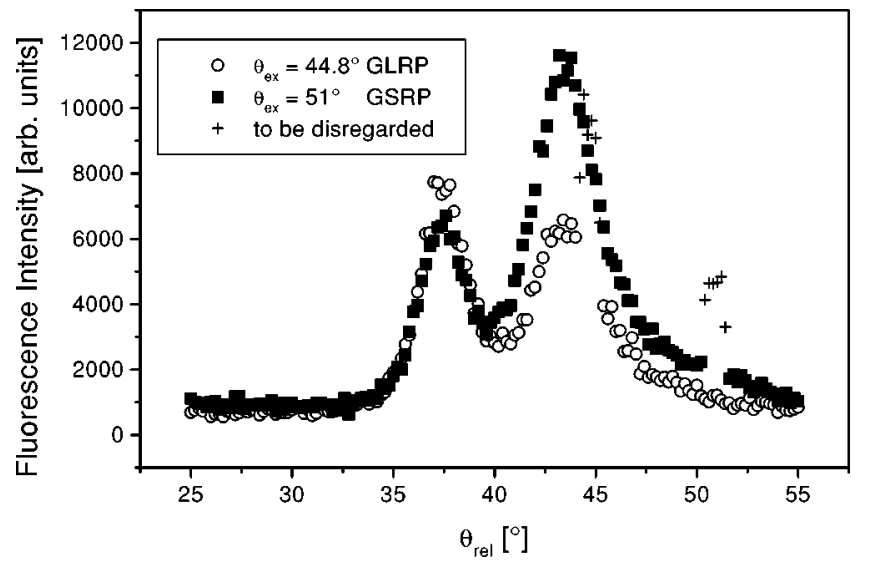

FIG. 9. Fluorescence intensity at $\lambda_{e m}=670 \mathrm{~nm}$ as a function of the emission angle $\theta_{e m}\left(=\theta_{r e l}\right)$ at $\psi_{e m}=90^{\circ}$ for two different excitation angles $\theta_{e x}$. The excitation wavelength equals $\lambda_{e x}$ $=632.8 \mathrm{~nm}$. When the specular reflection of the incoming beam coincides with the detection axis, there is some undesired stray light reaching the detector. These data points are drawn as crosses and should not be considered for the analysis.

excitation angles. Excitation of the GLRP (at $\lambda$ $=632.8 \mathrm{~nm}$ ) leads to an enhanced fluorescence emission mediated by the GLRP $\left(\theta_{\text {rel }}=37.5^{\circ}\right.$ at $\left.\lambda=670 \mathrm{~nm}\right)$. The same holds for the GSRP. The transfer from excitation to fluorescence energy is more effective between coupled resonances of equal type than between different types. This effect can be explained with the concept of the site-dependent variation of the excitation and emission probability as it was introduced in Sec. ID. In order to quantify this effect, the "Correlation number" $C$ is defined as

$$
C=\frac{I_{G L R P}^{G L R P} / I_{G S R P}^{G L R P}}{I_{G L R P}^{G S R P} / I_{G S R P}^{G S R P}} .
$$

$I$ denotes the maximum intensity of the peak as it is read from the plot and the superscript/subscript indicate whether the excitation/emission angle corresponds to the GLRP or GSRP coupled resonance. In the present case (Fig. 9) a correlation number of $C=2.12$ is found.

Some remarks must be made about the experimental errors. The final angular resolution of the detector leads to a smearing both in the direction of $\theta_{\text {rel }}$ (which will broaden the narrower peak at $37^{\circ}$ ) and in $\psi_{\text {tilt }}$, which will lead to a generally diminished effect as will be shown in the next section. The finite wavelength resolution of the detector has a similar effect. For these reasons, it can be assumed that the real correlation number is higher than the one that was determined from the experimental data.

Outside the resonance region, a significantly higher signal in the measurement than in the calculation is observed (compare the following section). This effect may be due to scattering effects by some roughness on the grating surface. This background will again lead to a reduction of the correlation number as found from the measurement. One may tend to eliminate this background by subtracting a constant number from the data, but this does not give satisfying results in the region around $\theta_{\text {rel }}=50^{\circ}$.

It is not meaningful to attempt to quantify all these effects, especially the error that is induced by scattering. Therefore, no error is assigned to the measured correlation. It should be kept in mind, though, that the measured correlation is too low.

\section{Detection in the plane of incidence: model calculations}

Calculations based on the two models introduced in Sec. II, assuming quickly rotating dipoles and fixed dipoles were performed. The calculated fluorescence intensities for both models are compared to the measured data in Fig. 10. A scaling factor was applied to the calculated data to obtain best correspondence to the measurement. Note that a scaling factor does not change the correlation as defined in Eq. (4.1)

Calculations based on both models predict a significant correlation. The correlation number that is found for the rotating dipoles is 1.79 , smaller than in the measurement $(C$


FIG. 10. The measured data for (a) $\theta_{e x}=44.8^{\circ}$ (excitation with the GLRP) and for (b) $\theta_{e x}=51^{\circ}$ (excitation with the GSRP) together with model calculations based on the model of fast rotating dipoles (narrow line) and fixed dipoles (broad line). 

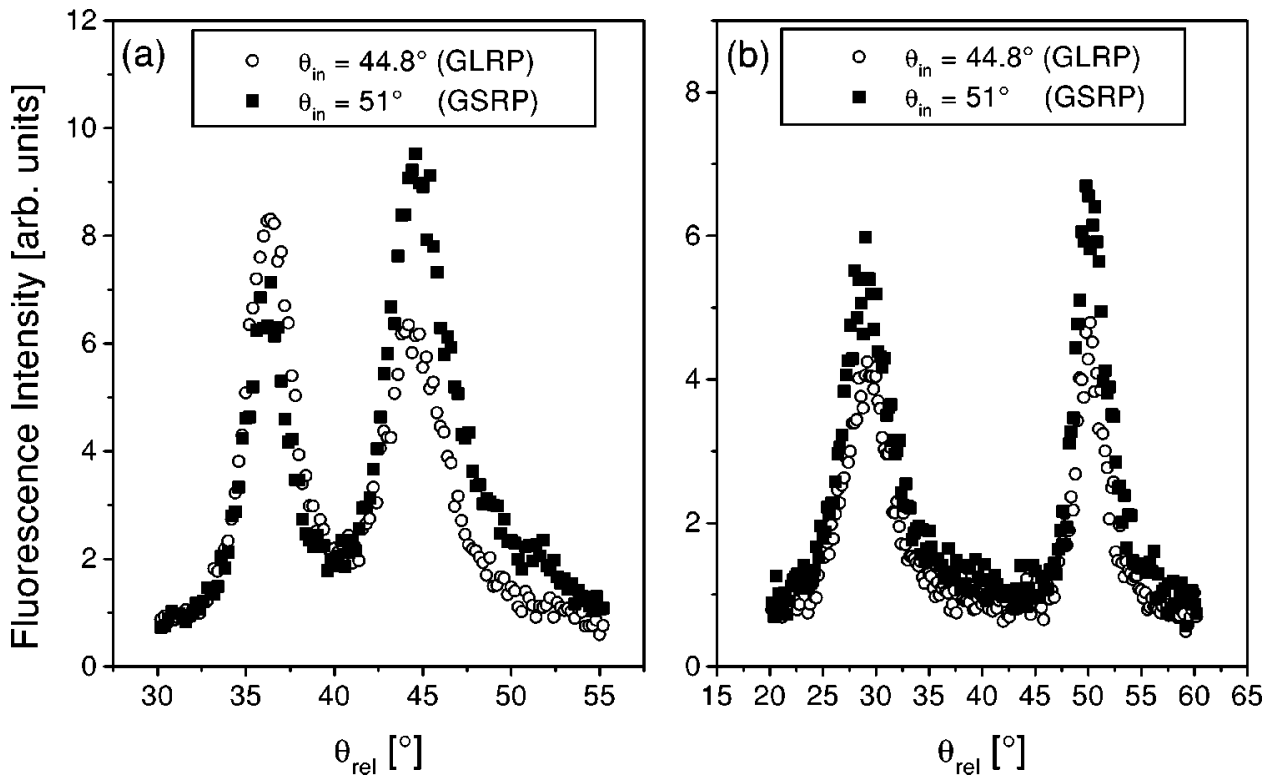

FIG. 11. Fluorescence intensity at (a) $\psi_{\text {tilt }}=1.6^{\circ}$ and at (b) $\psi_{\text {tilt }}=5.5^{\circ}$ for the two different angles of incidence corresponding to the excitation of the GLRP and the GSRP.
$=2.12$ ). The fixed dipoles on the other hand predict a correlation number of 2.93 , which is too high. Since the two averaging procedures can be regarded as extreme cases, it is not surprising that neither is able to give a perfect description of the measured data. Furthermore, the roughness of the sample induces some uncertainties in the grating profile as is determined from reflectivity measurements. Therefore, the intensity distributions as determined from the model calculations will have some uncertainties, too.

In conclusion, both averaging procedures predict a correlation that is of the same order of magnitude as the one observed experimentally. Due to the errors in the measurement and in the modeling, a quantitative evaluation is not possible. Still, the approximate agreement between experiment and theory gives a good insight into the underlying physics.

\section{Emission outside the plane of incidence}

Some more fluorescence data were recorded with the detector being positioned outside the plane of incidence $\left(\psi_{\text {tilt }}\right.$ $\neq 0^{\circ}$, therefore, $\left.\psi_{e m} \neq 90^{\circ}\right)$. These experiments track the resonance features involved in the outcoupling process in their evolution from coupled resonances (at $\psi_{\text {tilt }} \neq 0^{\circ}$ ) to free surface plasmons for higher $\psi_{\text {tilt }}$ (compare Figs. 4 and 5). It is expected that the correlation number for an outcoupling process that is mediated by free-surface plasmons will approach one (no correlation) due to the fact that there is no field localization. Figure 11 shows data measured with the detector positioned outside the plane of incidence by $\psi_{\text {tilt }}$ $=1.6^{\circ}$ and $\psi_{\text {tilt }}=5.5^{\circ}$.

The distance between the two peaks increases with increasing $\psi_{\text {tilt }}$ as it is expected from Fig. 4. While for small tilt angles $\left(\psi_{\text {tilt }}=1.6^{\circ}\right)$ these resonances in the transition regime from coupled to free-surface plasmons, the ones at higher tilt angles $\left(\psi_{\text {tilt }}=5.5^{\circ}\right)$ are sufficiently away from the photonic band gap and can therefore be regarded as freesurface plasmons.
For the emission recorded close to the plane of incidence $\left(\psi_{t i l t}=1.6^{\circ}\right)$, it can still be observed that the excitation of the chromophores via the GLRP leads to a more pronounced emission mediated by the GLRP while the excitation of the GSRP leads to an emission pattern for which the GSRP as an intermediate step is dominant. The correlation number as defined in Eq. (4.1) is 1.96, less than the 2.12 that were found in the measurement in the plane of incidence.

For $\psi_{t i l t}=5.5^{\circ}$, no difference in shape between the emission spectra upon excitation of the GLRP compared to the excitation of the GSRP can be seen. The correlation number is determined as 1.024 which is equal to 1 within the error.

This allows for the interpretation that only emission mediated by coupled resonances shows a site-selective emission in contrast to the process involving free-surface plasmons.

The correlation numbers that were obtained from several out-of-plane measurements, including the two that were just presented, are shown in Fig. 12. For comparison, correlation numbers that were obtained by calculations based on the two models are shown.

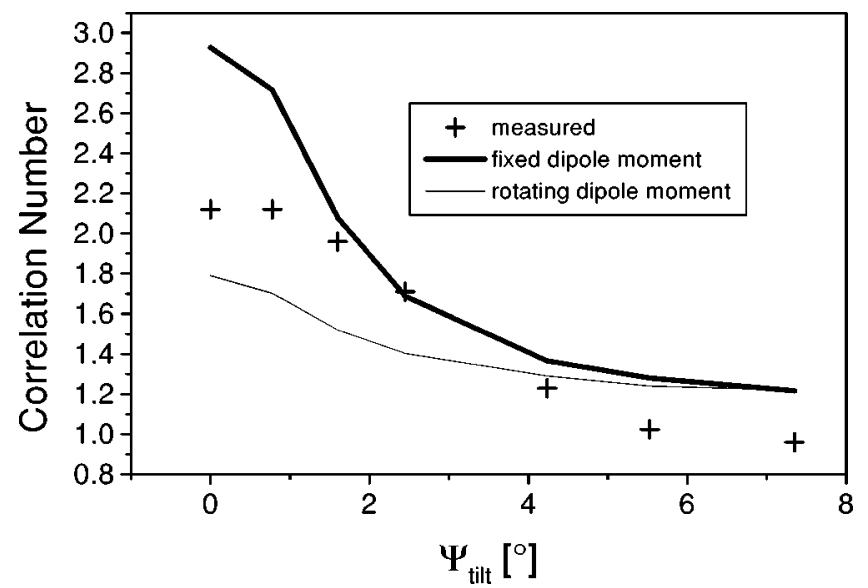

FIG. 12. Correlation numbers as a function of $\psi_{t i l t}$. Measured data are compared to model calculations. 
The measured correlation number shows a maximum when the detector is in the plane of incidence and decreases quickly when the detector is tilted out of the plane of incidence. At $\psi_{t i l t}=7.35^{\circ}$, the measured correlation number has reached one within the error of the measurement indicating freely propagating surface plasmons.

The model calculations assuming quickly rotating molecules (thin black line) yield significantly lower values than the ones measured in the plane of incidence, but a measurable correlation for strongly tilted detector axis is predicted that is not found in the measurement.

The modeled correlation numbers assuming fixed dipole moments (thick line) is higher than the measured data. It seems to decay more rapidly in the vicinity of $\psi_{t i l t}=0^{\circ}$ than in the measurement and there is a finite correlation predicted for $\psi_{t i l t}=7.35^{\circ}$ that is not found experimentally.

In conclusion, none of the averaging procedures is appropriate for a quantitative interpretation of the data. Nevertheless, both describe the reduced correlation upon transition from coupled resonances to freely propagating surface plasmons and predict the range of $\psi_{t i l t}$ where a significant correlation is observed approximately right. So, although some refinements seem to be necessary, the physical processes underlying the results of the measured fluorescence light that is coupled out with either a coupled resonance or a freely propagating surface plasmon as an intermediate step, are well reproduced by the presented theory.

\section{E. Selective bleaching}

In the previous section, there was considerable effort put in the elimination of bleaching effects. Nevertheless, the bleaching of chromophores in the localized fields of electromagnetic surface waves is another way to study the localization of the electrical fields of these resonances.

The photonic energy that is absorbed by a dye molecule may cause a photochemical reaction that transforms the dye into some nonfluorescent molecule. As a consequence, the fluorescence of a given sample will decrease with time under illumination. Obviously, when the chromophores are exposed to a spatially modulated electrical field pattern as it is generated by coupled surface resonances, the bleaching will mainly occur to the chromophores that are exposed to highfield intensities. This will lead to a modulated chromophore density across the grating with the same modulation period as the electrical field, $385 \mathrm{~nm}$ in the present case.

The existence of such a modulation can be proven by following the fluorescence intensity as a function of excitation and emission angle with time. Because the bleaching is relatively fast, only the two excitation angles (GLRP: $\theta_{e x}$ $=44.8^{\circ}$, GSRP: $\theta_{e x}=51^{\circ}$ ) and two emission angles (GLRP: $\theta_{e m}=37.5^{\circ}$, GSRP: $\theta_{e m}=43.5^{\circ}$ ) are chosen that assure maximum coupling of (incident or emitted) light to the coupled surface resonances. These four intensities are measured while the sample is illuminated continuously under a fixed angle $\theta_{\text {bleach }}$. When $\theta_{\text {bleach }}$ is chosen to coincide with the maximum excitation of one resonance, the corresponding emission can be measured in situ during the bleaching process. The measurement of the emission excited by the other

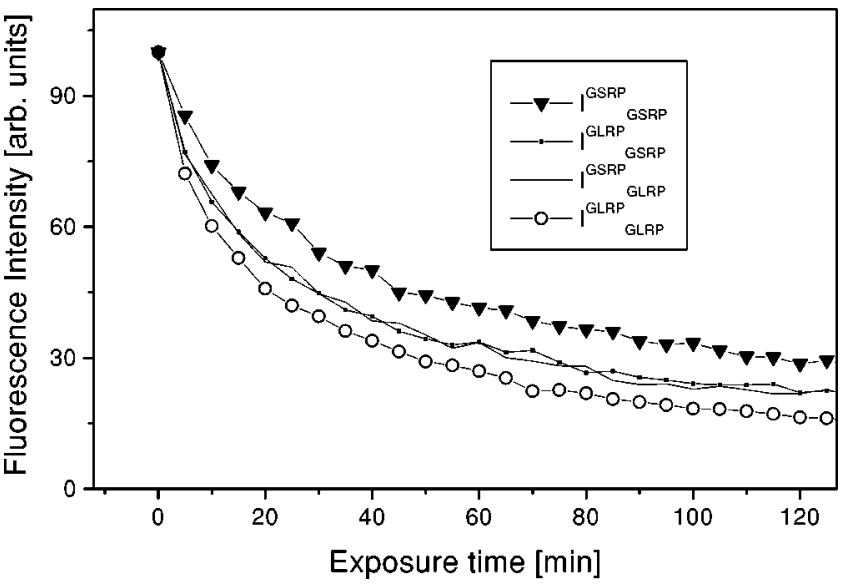

FIG. 13. Evolution of the intensities $I_{G L R P}^{G L R P}, I_{G S R P}^{G L R P}, I_{G L R P}^{G S R P}$, $I_{G S R P}^{G S R P}$, during illumination in the GLRP. The curves were normalized to a starting value of 100 to allow better comparison.

resonance requires a short interruption of the bleaching process as well as illumination at a "wrong" excitation angle, resulting in an unwanted destruction of dye molecules. Bleaching intervals of five minutes were chosen with data acquisition times of ten seconds per point to minimize the effect of bleaching at the wrong angle. Figure 13 shows the four peak intensities for bleaching under $\theta_{\text {bleach }}=44.8^{\circ}$ (excitation of the GLRP) with $\lambda_{e x}=632.8 \mathrm{~nm}$. It is clearly seen that the decay of $I_{G L R P}^{G L R P}$ is the fastest, followed by $I_{G S R P}^{G L R P}$ and $I_{G L R P}^{G S R P}$, which are approximately equal. $I_{G S R P}^{G S R P}$ exhibits the slowest decay. $I$ denotes the fluorescence intensity, the superscript the excitation, and the subscript the emission direction.

This behavior can be explained qualitatively if one analyzes the molecules involved in the various fluorescence processes:

(1) $I_{G L R P}^{G L R P}$ mainly involves the molecules in the intensity maxima of the GLRP.

(2) $I_{G S R P}^{G S R P}$ mainly involves the molecules in the intensity maxima of the GSRP.

(3) $I_{G S R P}^{G L R P}$ and $I_{G L R P}^{G S R P}$ mainly involves the molecules that sense significant field strength of both coupled resonances, this is right in the slopes of the intensity distributions in Fig. 6.

Because the bleaching rate is proportional to the local electric-field strength, the different bleaching rates that are observed in Fig. 13 can be understood based on the assumption that the chromophores placed in the intensity maxima of the GLRP will be most effectively bleached. Indeed, the bleaching beam writes a modulation in chromophore density and this effect can be tracked by observation of the fluorescence intensities at the experimental geometries specified above.

\section{CONCLUSION AND OUTLOOK}

It has been demonstrated experimentally that the localization of the near field of coupled surface resonances on me- 
tallic gratings leads to spatially inhomogenous excitation and emission properties of dye molecules near the surface. A theoretical model allows for a qualitative description of these effects, but due to errors both in the model and in the measurement, a full quantitative description is not possible. Outcoupling mediated by free-surface plasmons does not show spatial selectivity. These results are an experimental verification of the theoretically predicted localization of the electrical field of coupled surface resonances as opposed to normal surface plasmons.

Additionally, the spatially inhomogenous bleaching rate resulting from the localization of the coupled resonances was demonstrated experimentally.

In conclusion, an experimental verification of the theoretically predicted form of the electromagnetic fields of coupled surface resonances is given. This allows for a well-defined manipulation of chromophores on metal surfaces.

It would be very interesting to undertake similar measurements with freely rotating chromophores as opposed to fixed ones and check if the theoretically expected reduction of the correlation upon enhanced mobility can be observed. The search for spatial inhomogenities of lifetimes and spectral properties due to coupled surface waves would be another interesting topic.
${ }^{1}$ H. Raether, Surface Plasmons (Springer, Berlin, 1988).

${ }^{2}$ R. W. Ritchie, E. T. Arakawa, J. J. Cowan, and R. N. Hamm, Phys. Rev. Lett. 21, 1530 (1968).

${ }^{3}$ W. L. Barnes, T. W. Preist, S. C. Kitson, J. R. Sambles, N. P. K. Cotter, and D. J. Nash, Phys. Rev. B 51, 11164 (1995).

${ }^{4}$ D. Sarid, Phys. Rev. Lett. 47, 1927 (1981).

${ }^{5}$ T. W. Preist, J. B. Harris, N. P. Wanstall, and J. R. Sambles, J. Mod. Opt. 44, 1073 (1997).

${ }^{6}$ Y. M. Gerbshtein, I. A. Merkulov, and D. N. Mirlin, Pis'ma Zh. Éksp. Teor. Fiz. 22, 80 (1975) [JETP Lett. 22, 35 (1976)].

${ }^{7}$ W. H. Weber and C. F. Eagen, Opt. Lett. 4, 236 (1979).

${ }^{8}$ R. E. Benner, R. Dornhaus, and R. K. Chang, Opt. Commun. 30, 145 (1979).

${ }^{9}$ I. Pockrand, A. Brillante, and D. Möbius, Chem. Phys. Lett. 69, 499 (1979).

${ }^{10}$ H. Knobloch, H. Brunner, A. Leitner, F. Aussenegg, and W. Knoll, J. Chem. Phys. 98, 10093 (1993).

${ }^{11}$ S. C. Kitson, W. L. Barnes, J. R. Sambles, and N. P. K. Cotter, J. Mod. Opt. 43, 573 (1996).

${ }^{12}$ P. Andrew, S. C. Kitson, and W. L. Barnes, J. Mod. Opt. 44, 395 (1997).

${ }^{13}$ A. Hinchcliffe and R. W. Munn, Molecular Electromagnetism
(Wiley, New York, 1985).

${ }^{14}$ R. R. Chance, A. Prock, and R. Silbey, Adv. Chem. Phys. 37, 1 (1978).

${ }^{15}$ J. E. Sipe, Surf. Sci. 105, 489 (1981).

${ }^{16}$ G. W. Ford and W. H. Weber, Phys. Rep. 113, 195 (1984).

${ }^{17}$ W. L. Barnes, J. Mod. Opt. 45, 661 (1998).

${ }^{18}$ K. H. Drexhage, Sci. Am. 222, 108 (1970).

${ }^{19}$ A. Adams et al., Phys. Rev. B 25, 3457 (1982).

${ }^{20}$ W. Knoll, M. R. Philpott, J. D. Swalen, and A. Girlando, J. Chem. Phys. 75, 4795 (1981).

${ }^{21}$ S. C. Kitson, W. L. Barnes, and J. R. Sambles, Opt. Commun. 122, 4 (1996).

${ }^{22}$ S. C. Kitson, W. L. Barnes, and J. R. Sambles, Phys. Rev. B 52, 11441 (1995).

${ }^{23}$ W. L. Barnes, T. W. Preist, S. C. Kitson, and J. R. Sambles, Phys. Rev. B 54, 6227 (1996).

${ }^{24}$ X. Mai, R. Moshrefzadeh, U. J. Gibson, G. I. Stegeman, and C. T. Seaton, Appl. Opt. 24, 3155 (1985).

${ }^{25}$ R. A. Watts, J. R. Sambles, M. C. Hutley, T. W. Preist, and C. R. Lawrence, Nanotechnology 8, 35 (1997).

${ }^{26}$ M. Kreiter, J. Oster, R. Sambles, S. Herminghaus, S. MittlerNeher, and W. Knoll, Opt. Commun. 168, 117 (1999). 